\title{
Quelques réflexions sur la mécanique de l'écoulement des mélanges d'eau et d'air
}

\section{Notes on the flow mechanics of water-air mixtures}

Lans le cas des éconlements mixtes d'ean et d'air, le coefficient de rugosité n'est plus une constante comme dans l'Hydraulique classique. Cependant, la valeur de ce coefficient tendrait vers nne constante, uniberselle, au fur et it mesure de l'accroissement de la concentration en air.

Suggestion d'une nouvelle formule liant la concentration en rair allx caractéristiques hydrauliques dn canal.

I. - Dépendance du coefficient de Rugosité $n$ VIS-A-VIS DE LA « CONCENTRATION EN AIR $\gg 1-p$.

Le coefficient de rugosité $n$ garde, comme on sait, une valeur sensiblement constante dans les écoulements d'eau seule dans un canal donné. Il n'en est plus de même dans les écoulements de mélange d'eau et d'air. Il a été expérimentalement constaté que le coefficient de rugosité $n$ dépendait de la concentration en air $(1-0)$. La formule employée est :

$$
\mathrm{V}=\frac{1}{n} \mathrm{R}^{2 / 3} \mathrm{I}^{1 / 2}
$$

avec :

$\mathrm{V}=$ vitesse moyenne du mélange d'eau et d'air,

\begin{abstract}
For the fiow of water-air mirlures the roefficient of frictional resistance is no longer a constant expression as il is in classical hlydraulics. Honever its magnitude seems to tend towards a constant as the eonentration of air in the emulsion increases. A new formula is suggested relating air concentration to the hijdraulic characteristics of the canal.
\end{abstract}

$\mathrm{R}=$ rayon hydraulique du mélange d'eau et d'air,

$\mathrm{I}=$ pente du fond du canal.

Dans le rapport "Entrainement of Air in flowing water and technical problems connected with it " présenté par M. V. JEvinsvich el moimême au $V^{a}$ Congrès de l'A.I.R.H. à Minneapolis en septembre 1953 , a été émise l'opinion que le coefficient de rugosité dépendait de la concentration en eau au fond; il diminuait abec l'accroissement de la concentration en air au fond 1 - ?

Il me paraît personnellement, après nouvelle étude, plus commode de relier le coefficient de rugosité $n$ non pas à la concentration sur le fond mais à la concentration moyenne. Ce procédé, tout empirique qu'il soit, serait justifié à l'heure actuelle, et faute de pouvoir faire mieux, par sa commodité dans les opérations de calcul. 
Nous avons représenté sur le graphique cidessous les résultats de mesure sur 9 ouvrages de rugosité très différente et notamment:

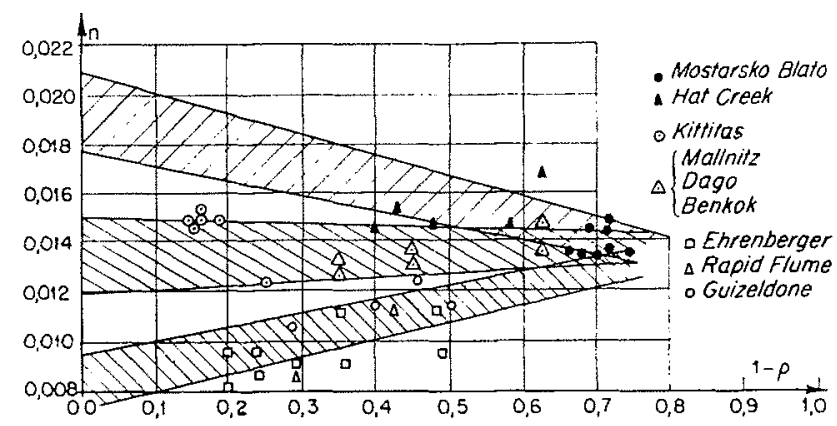

\section{2 canaux très rugueux :}

- Hat Creek (U.S.A.),

- Mostarsko Blato (Yougoslavie).

4 canaux de rugosité moyenne (béton normal):

- Kittitas (U.S.A.),

- Dago (Indonésie),

- Mallnitz (Autriche),

- Bankok (Indonésie).

3 canaux très lisses (bois) :

- d'Ehrenberger au Laboratoire de Vienne,

- Rapid Flume (U.S.A.),

- Guizeldone (U.R.S.S.).

L'analyse du graphique fait ressortir une intéressante propriété des écoulements de mélanges d'eau et d'air, à savoir : le coefficient de rugosité $n$ tend, avec l'accroissement de la concentration en air $(1-\mathrm{p})$, vers une valeur universelle, constante, indépendante du coefficient de rugosité $n_{0}$ afférent à l'eau scule (c'est-à-dire $p=1$ ). Avec l'augmentation du pourcentage d'air, le coefficient de rugosité tend vers une valeur propre au fluide-mélange. Pour $1-\rho=0,80$ environ, le coefficient de rugosité s'élève, pour canaux de toute rugosité, à $n=0,0135$ environ $\left({ }^{*}\right)$.

Ce fait évoque une analogie avec les écoulements de mélange d'eau et de matériaux fins en suspension : là aussi avec l'accroissement de la concentration, le coefficient de rugosité tend vers une constante universelle.

(*) Je me fais un plaisir de rendre ici hommage à mon collègue, M. S. Bruk, Ingénieur au Laboratoire d'Avala (Yougoslavie), avec qui, dans une discussion privee, nous sommes parvenus intuitivement à cetta idée.
Sur la base du graphique ci-dessus, il paraît possible de recommander la formule suivante :

$$
n=0,017-0,25 n_{0}+1,25 \rho n_{0}-0,017 \text { ? }
$$

avec $:=$ concentration en eau du mélange.

\section{II. - DéPENDANCE DU GOEFFICIENT O VIS-A-vis DES CaRACTÉRISTIQUeS HYDRAULIQUES DU CANAL.}

Dans notre rapport mentionné ci-dessus, M. V. JEvDIEVIch et moi-même avions proposé la formule :

$$
\frac{1-\rho}{\varphi}=\mathrm{A} \cdot \mathrm{F} \cdot \varphi \cdot \alpha_{0}
$$

de structure identique à celle de S. HALL $\left.{ }^{\star \star}\right)$. Dans cette formule, les symboles ont les significations suivantes :

$A=$ constante universelle,

$\mathrm{F}=\frac{\mathrm{V}^{2}}{g \mathrm{R}}=$ carré du nombre de Froude,

$\varphi=\frac{n_{0}}{\mathrm{R}^{1 / 6}} \sqrt{g}=$ coefficient de perte par frottement.

$\alpha_{a}=\frac{\int_{0}^{h} \rho \mathrm{V}^{3} d h}{\rho_{m} \mathrm{~V}_{m}^{3} h}=$ coefficient tenant compte de la répartition des vitesses et de la concentration en eau.

$h=$ profondeur d'eau,

l'indice « $m »$ indiquant que la grandeur est une valeur moyenne.

La formule (3) était, tout comme la formule de Hall et les autres formules publiées dans le passé, valable uniquement pour une zone de variation de la vitesse moyenne $V$ et du rayon hydraulique $\mathrm{R}$ correspondant aux conditions d'expérimentation, c'est-à-dire approximativement pour :

$$
\begin{aligned}
& \mathrm{V}=15 \text { à } 30 \mathrm{~m} / \mathrm{s}, \\
& \mathrm{R}=0,07 \text { à } 0,30 \mathrm{~m} .
\end{aligned}
$$

Il est, à priori, évident que la formule (3) ne peut s'appliquer pour toutes vitesses, car il est

\footnotetext{
(* *) Transactions et A.S.C.E., vol. 108, 1943.
} 
connu qu'au-dessous d'une vitesse $V=V_{0}$ (de l'ordre de 2 à $5 \mathrm{~m} / \mathrm{s}$ ), il n'est pas possible de s'écarter de $\rho=1$, aussi élevé que soit $F$. En admettant la formule (3) « pleinement » valable pour $\mathrm{V} \geqslant \mathrm{V}_{1}\left(\mathrm{~V}_{1}\right.$ de l'ordre de 16 à $\left.20 \mathrm{~m} / \mathrm{s}\right)$, il est évident que pour la zone de vitesses entre $V_{0}$ et $V_{1}$, la formule (3) n'est qu'approchée et d'autant moins que $V$ se rapproche de $V_{0}$.

Nous estimons personnellement possible d'essayer de lier le rapport $(1-0) / s$ non pas à $V^{2}$, mais à $\left(V-V_{0}\right)^{2}$.

$V_{0}$ est la «vilesse de commencement d'entrainement de l'air dans l'eau $\gg$ que nous croyons devoir lier à la rugosité $n_{0}$ et à la profondeur $h$.

$$
\mathrm{V}_{0}=\int\left(n_{0}, h\right)
$$

Cette relation devrait faire l'objet, avant tout, de recherches expérimentales.

La formule recherchée aurait l'aspect suivant:

$$
\frac{1-\rho}{?}=\mathrm{K} \cdot \hat{\mathrm{Q}} \frac{\left(\mathrm{V}-\mathrm{V}_{0}\right)}{g h}
$$

aree:

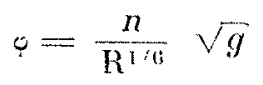

où $\mathrm{K}$ est une constante expérimentale, et $n$ un coefficient de rugosité délerminé par la formule (2).

Léon Levis, Ingénieur E.I.H., boctenx is sciences techniques.

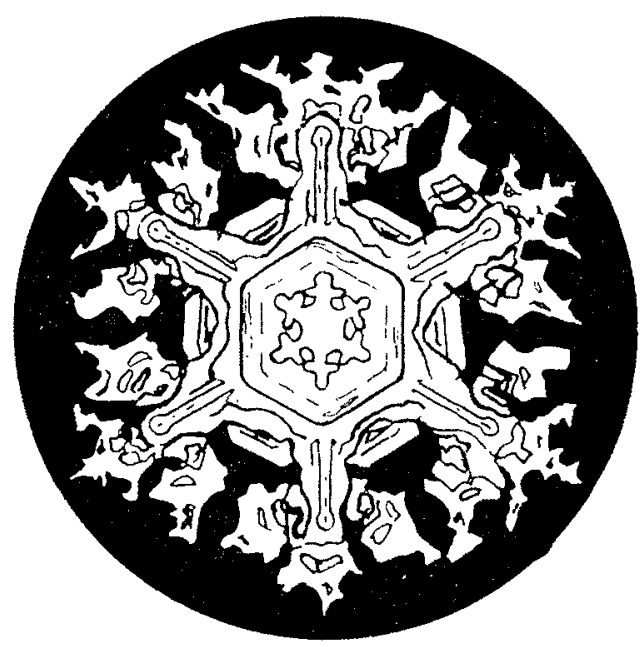

